\title{
MEASURING ETHNIC STRATIFICATION AND ITS EFFECT ON TRUST IN AFrICA
}

\author{
Noémie Zurlinden ${ }^{1}$ \\ Joint work with Roland Hodler ${ }^{1}$, \\ Sorawoot Srisuma ${ }^{2}$ and Alberto Vesperoni ${ }^{3}$ \\ ${ }^{1}$ University of St.Gallen, Switzerland \\ ${ }^{2}$ University of Surrey, UK \\ ${ }^{3}$ Alpen-Adria University Klagenfurt, Austria
}

CSAE Conference 2019

March 17-19, 2019 


\section{ETHNOLINGUISTIC DIFFERENCES}
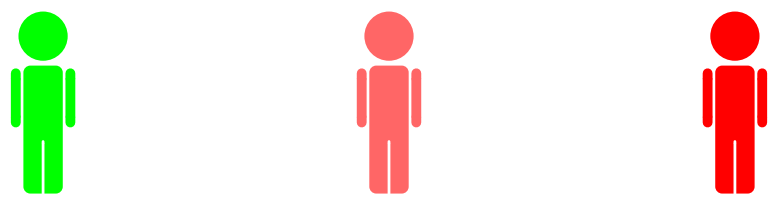


\section{ECONOMIC DIFFERENCES}
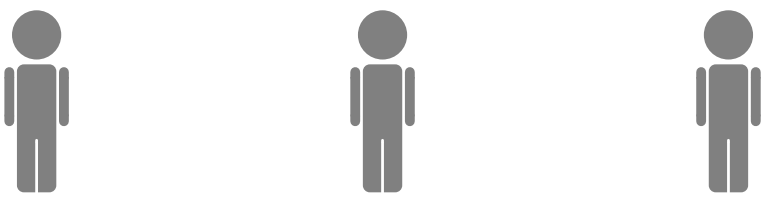

$\$$

$\$ \$$

$\$ \$ \$$ 


\section{ETHNIC STRATIFICATION}

COMBINING ETHNOLINGUISTIC AND ECONOMIC DIFFERENCES
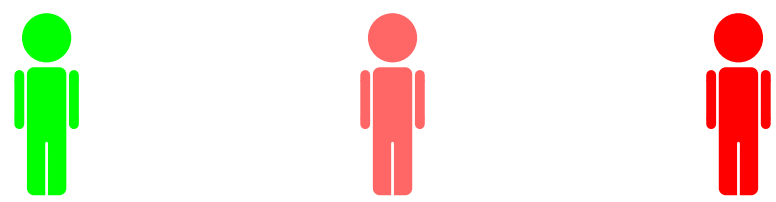

$\$$

$\$ \$$

$\$ \$ \$$ 


\section{ETHNIC STRATIFICATION}

\section{TAKING INTO ACCOUNT ETHNOLINGUISTIC DISTANCES}
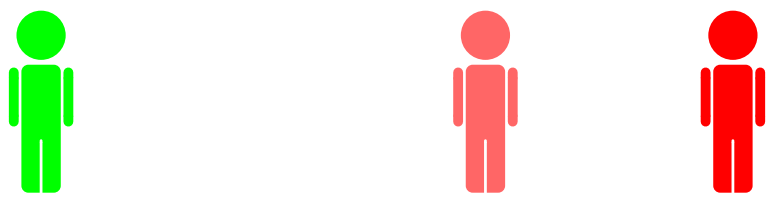


\section{ETHNIC STRATIFICATION}
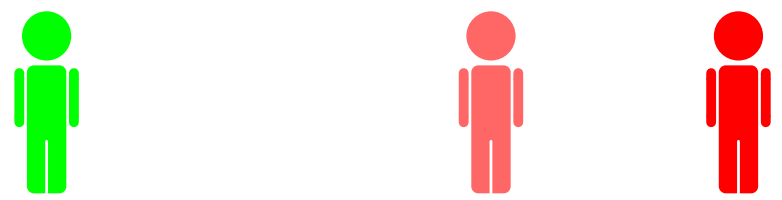

$\$$

$\$ \$$

$\$ \$ \$$

- Alienation (or mistrust) between two individuals depends on their economic and ethnolinguistic distances

- Ethnic STRATification: Extent to which the hierarchy in socio-economic positions follows ethnic lines 


\section{ThE PAPER IN A NUTSHELL}

- Theoretical Part: Index of ethnic stratification

- EMPIRICAl PART: We show that ethnic stratification on the local level in Africa is related to lower trust towards individuals and institutions 


\section{LITERATURE}

- Theoretical literature on inequality AND DiVersity MEASUREMENT

- Review on inequality and diversity measurement: Chakravarty (2015)

- Gini (e.g., Shorrocks 1980), multivariate Gini (e.g., Banerjee 2010)

- Univariate fractionalization (Bossert et al. 2011)

- Polarization (e.g., Esteban and Ray 1994; Reynal-Querol and Motalvo 2005; Permanyer 2012)

- Empirical literatures on BetWeEn-GRoup inEQUALity AND TRUST

- Literature on POLITiCO-ECONOMiC CONSEQUenCES OF BETWEEN-GROUP INEQUALITY: e.g., low GDP per capita (Alesina et al. 2016), low public good provision (Baldwin and Huber 2010), civil conflict (Gubler and Selway 2012, Cederman et al. 2011, Guariso and Rogall 2016, Huber and Mayoral 2014)

- Literature on Determinants of trust in Africa And ELSEWHERE: e.g., between-group inequality (Tesei 2015), historical experiences (Nunn and Wantchekon 2011), conflict (Rohner et al. 2013), protests (Sangnier and Zylberberg 2017), ethnic diversity (Robinson 2017) 


\section{THEORETICAL PART: INDEX OF ETHNIC STRATIFICATION}




\section{INDEX OF ETHNIC STRATIFICATION}

- Population $P$ of size $m>0$

- Distance between wealth levels $w_{i}$ and $w_{j}:\left|w_{i}-w_{j}\right|$

- Distance between ethnicities $e_{i}$ and $e_{j}: \lambda\left(e_{i}, e_{j}\right)$

\section{INDEX OF ETHNIC STRATIFICATION}

$$
S(f, \lambda):=\frac{1}{m^{2}} \int_{i \in P} \int_{j \in P} \lambda\left(e_{i}, e_{j}\right)\left|w_{i}-w_{j}\right| d j d i
$$

- Member of general class of measures of expected alienation between a randomly selected pair of individuals

- Axiomatic Approach: We show that the proposed index fulfills axioms that are desirable properties of a measure of ethnic stratification 


\section{GENERALIZATION OF BETWEEN-GROUP INEQUALITY}

Village A

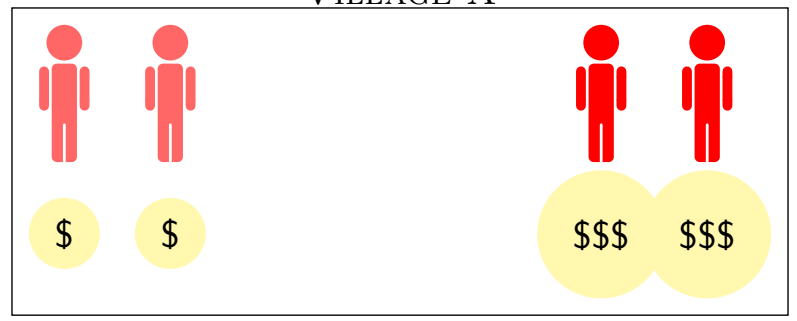

Village B

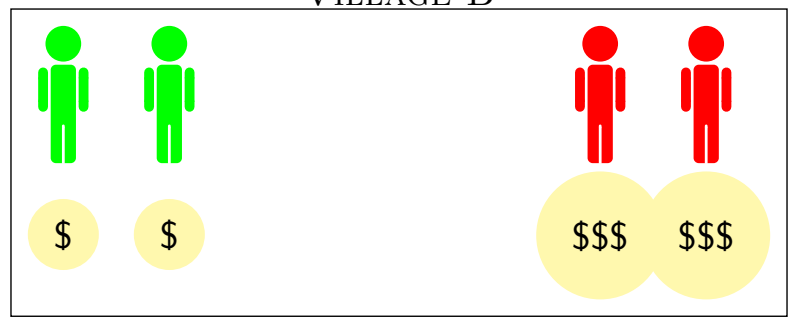




\section{DECOMPOSITION OF ETHNIC STRATIFICATION}

- Average wealth:

$\mu(w):=\frac{1}{m} \int_{i \in P} w_{i} d i$

- Gini coefficient of inequality:

$G(w):=\frac{1}{2 \mu\left(\gamma_{f}\right) m^{2}} \int_{i \in P} \int_{j \in P}\left|w_{i}-w_{j}\right| d j d i$

- Ethnic fractionalization:

$$
F(e):=\frac{1}{m^{2}} \int_{i \in P} \int_{j \in P} \lambda\left(e_{i}, e_{j}\right) d j d i
$$




\section{SCALE VS. CO-DIRECTIONALITY}

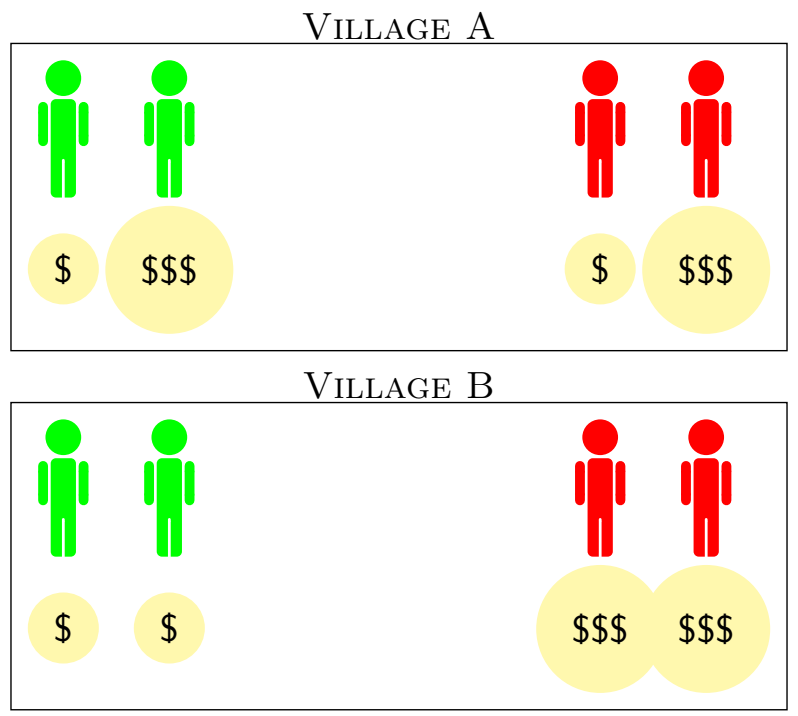




\section{EMPIRICAL PART: LOCAL ETHNIC STRATIFICATION AND TRUST IN AFRICA}




\section{EMPIRICAL PART}

- We measure ethnic stratification on the town and village level in Africa and investigate its effect on trust

- DAtA: Afrobarometer survey, rounds 5 (2011-2013) and 3

(2005-2006)

- Information on assets, language, ethnicity, trust

- Data geocoded by Aiddata 


\section{COMPUTING WEALTH DISTANCES}

Asset-BASED WEALTh INDEX:

- First principal component (Principal components analysis)

- Calculated for each survey (country and survey round) separately

- Assets round 5: Radio, television, mobile phone, motorcycle/car, types of water source, toilet, house and roof

- Assets round 3: Radio, television, bicycle, motorcycle and car 


\section{DERIVING ETHNOLINGUISTIC DISTANCES}

Match Afrobarometer languages and ethnicities to ETHNOLOGUE

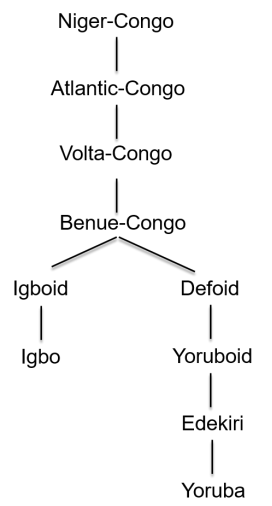

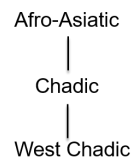

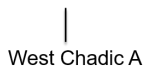

$\left.\right|_{\text {West Chadic A.1 }}$

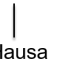

Figure 1: Language trees 


\section{DERIVING ETHNOLINGUISTIC DISTANCES}

Match Afrobarometer languages and ethnicities to ETHNOLOGUE

EthnOLINGUistic

DISTANCES BETWEEN

INDIVIDUALS: Distance measure by Putterman and Weil (2010):

$$
\lambda_{i, j}=1-\sqrt{\frac{2 t_{i j}}{T_{i}+T_{j}}}
$$

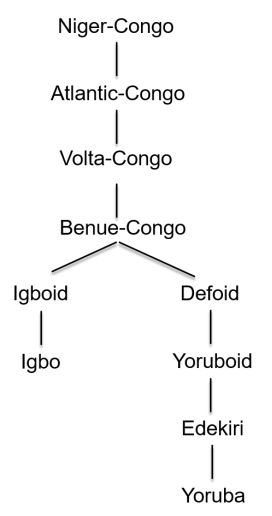

Afro-Asiatic
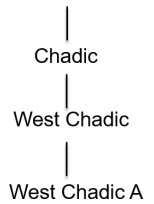

West Chadic A.1

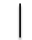

Hausa

Figure 1: Language trees 


\section{FINAL SAMPLE ROUND 5}

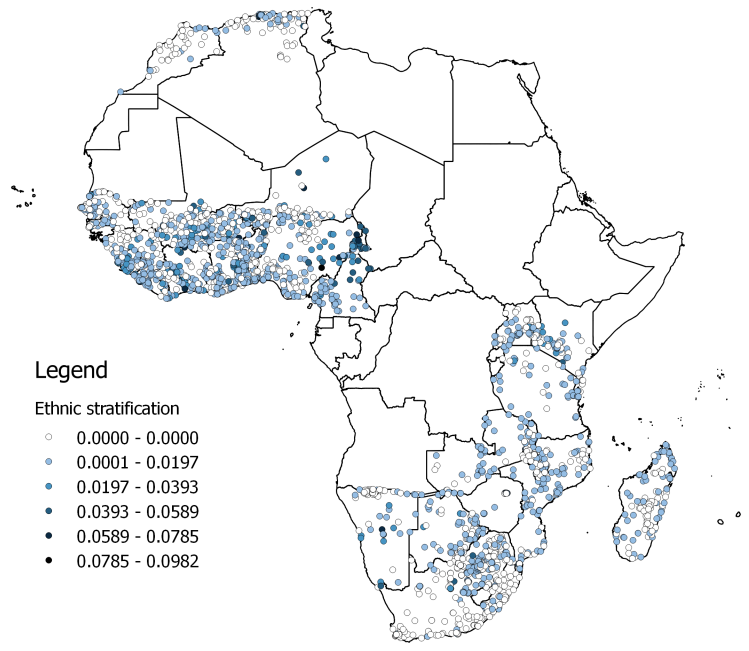




\section{MEASURING TRUst}

- Round 5: How much do you trust

- your relatives?

- your neighbors?

- other people you know?

- your metropolitan, municipal or district assembly?

- Round 3: How much do you trust

- people from your own ethnic group?

- people from other ethnic groups?

- We build indicator variables that equal one if the respondent answers "a lot" or "somewhat", and zero if she answers "just a little" or "not at all".

- Trust differential $=1$ if trust in own ethnic group $>$ trust in other ethnic groups 


\section{SUMMARY STATISTICS}

\begin{tabular}{lccccc}
\hline \hline Variable & Obs. & Mean & Std.Dev. & Min. & Max. \\
\hline Round 5: & & & & & \\
\hline Trust in relatives & 21,544 & 0.826 & 0.379 & 0 & 1 \\
Trust in neighbors & 21,521 & 0.607 & 0.488 & 0 & 1 \\
Trust in other acquaintances & 21,475 & 0.416 & 0.493 & 0 & 1 \\
Trust in local assembly & 19,236 & 0.493 & 0.500 & 0 & 1 \\
Ethnic stratification (S) & 21,579 & 0.007 & 0.013 & 0 & 0.098 \\
Fractionalization (F) & 21,579 & 0.103 & 0.147 & 0 & 0.696 \\
Wealth inequality (G) & 21,579 & 0.083 & 0.047 & 0 & 0.390 \\
Average wealth $(\mu)$ & 21,579 & 0.341 & 0.069 & 0.094 & 0.738 \\
\hline Round 3: & & & & & \\
Trust in own ethnic group & 11,833 & 0.550 & 0.498 & 0 & 1 \\
Trust in other ethnic group & 11,710 & 0.420 & 0.494 & 0 & 1 \\
Higher trust in own group & 11,683 & 0.263 & 0.440 & 0 & 1 \\
Ethnic stratification (S) & 11,860 & 0.013 & 0.022 & 0 & 0.142 \\
Fractionalization (F) & 11,860 & 0.101 & 0.149 & 0 & 0.639 \\
Wealth inequality (G) & 11,860 & 0.252 & 0.103 & 0 & 0.750 \\
Average wealth $(\mu)$ & 11,860 & 0.202 & 0.058 & 0 & 0.551 \\
\hline \hline
\end{tabular}




\section{SPECIFICATIONS}

$$
\text { Trust }_{i v c e}=\beta S_{v c}+\theta X_{i v c e}+F E_{c}+F E_{e}+\epsilon_{i v c e}
$$

- Trust ivce: $_{\text {: }}$ trust indicator for respondent $i$ living in town / village $v$ of country $c$ and belonging to ethnolinguistic group $e$

- $S_{v c}$ : ethnic stratification in town / village $v$

- $X_{\text {ivce }}$ : control variables (wealth, age, age squared, religion, education, urban/rural)

- $F E_{c}$ : country fixed effects

- $F E_{e}$ : ethnolinguistic group fixed effects

$$
\text { Trust }_{i v c e}=\beta S_{v c}+\gamma F_{v c}+\delta G_{v c}+\psi \mu_{v c}+\theta X_{i v c e}+F E_{c}+F E_{e}+\epsilon_{i v c e}
$$

- $F_{v c}$ : local ethnic fractionalization

- $G_{v c}$ : local wealth inequality (Gini)

- $\mu_{v c}$ : local average wealth

We estimate linear probability models. Standard errors are clustered at the level of ADM1 regions and ethnolinguistic groups. 


\section{ETHNIC STRATIFICATION AND TRUST IN INDIVIDUALS}

\section{AND INSTITUTIONS}

\begin{tabular}{lcccc}
\hline \hline & $(1)$ & $(2)$ & $(3)$ & $(4)$ \\
\hline Dep. variable: & $\begin{array}{c}\text { Trust in } \\
\text { relatives }\end{array}$ & $\begin{array}{c}\text { Trust in } \\
\text { neighbors }\end{array}$ & $\begin{array}{c}\text { Trust in other } \\
\text { acquaintances }\end{array}$ & $\begin{array}{c}\text { Trust in } \\
\text { local assembly }\end{array}$ \\
\hline Stratification & $-1.30^{* *}$ & $-2.19^{* * *}$ & $-1.73^{* * *}$ & $-1.31^{* *}$ \\
& $(0.52)$ & $(0.44)$ & $(0.34)$ & $(0.55)$ \\
$R^{2}$ & 0.16 & 0.18 & 0.12 & 0.13 \\
\hline Stratification & $-1.64^{*}$ & $-2.09^{* * *}$ & $-2.02^{* * *}$ & $-1.94^{* * *}$ \\
Fractionalization & $(0.94)$ & $(0.78)$ & $(0.69)$ & $(0.70)$ \\
& 0.07 & 0.03 & 0.06 & 0.09 \\
Gini & $(0.07)$ & $(0.07)$ & $(0.07)$ & $(0.07)$ \\
& -0.09 & -0.09 & -0.03 & 0.01 \\
Average wealth & $(0.09)$ & $(0.11)$ & $(0.12)$ & $(0.17)$ \\
& $-0.24 * * *$ & $-0.51^{* * *}$ & $-0.40 * * *$ & $-0.36 * *$ \\
$R^{2}$ & $(0.08)$ & $(0.14)$ & $(0.14)$ & $(0.15)$ \\
\hline Observations & 0.16 & 0.19 & 0.12 & 0.13 \\
\hline \hline
\end{tabular}

Notes: $* * *, * *, *$ indicate significance at the 1,5 and $10 \%$-level, respectively. 


\section{ETHNIC STRATIFICATION AND TRUST IN OWN/OTHER ETHNIC GROUPS}

\begin{tabular}{lccc}
\hline \hline & $(1)$ & $(2)$ & $(3)$ \\
\hline Dep. variable: & $\begin{array}{c}\text { Trust in own } \\
\text { ethnic group }\end{array}$ & $\begin{array}{c}\text { Trust in other } \\
\text { ethnic group }\end{array}$ & $\begin{array}{c}\text { Higher trust in } \\
\text { own ethnic group }\end{array}$ \\
\hline Stratification & 0.23 & -0.01 & -0.14 \\
$R^{2}$ & $(0.43)$ & $(0.43)$ & $(0.28)$ \\
\hline Stratification & 0.14 & 0.13 & 0.07 \\
Fractionalization & 0.28 & -0.16 & $0.99 *$ \\
& $(0.57)$ & $(0.72)$ & $(0.51)$ \\
Gini & 0.00 & 0.05 & $-0.18^{* *}$ \\
& $(0.10)$ & $(0.12)$ & $(0.08)$ \\
Average wealth & 0.00 & -0.02 & -0.07 \\
& $(0.07)$ & $(0.07)$ & $(0.07)$ \\
$R^{2}$ & -0.15 & -0.19 & -0.07 \\
Observations & $(0.15)$ & $(0.17)$ & $(0.14)$ \\
\hline \hline
\end{tabular}

Notes: $* * *, * *, *$ indicate significance at the 1,5 and $10 \%$-level, respectively. 


\section{CONCLUSION}

- We propose a new index of ethnic stratification based on both economic and ethnic distances between pairs of individuals.

- We calculate ethnic stratification on the town and village level in 26 African countries.

- Findings: Higher local ethnic stratification...

- decreases trust in people an individual knows and in local political institutions.

- makes it more likely that respondents trust people from their own ethnic group more than people from other ethnic groups. 


\section{Appendix}




\section{FINAL SAMPLE ROUND 3}

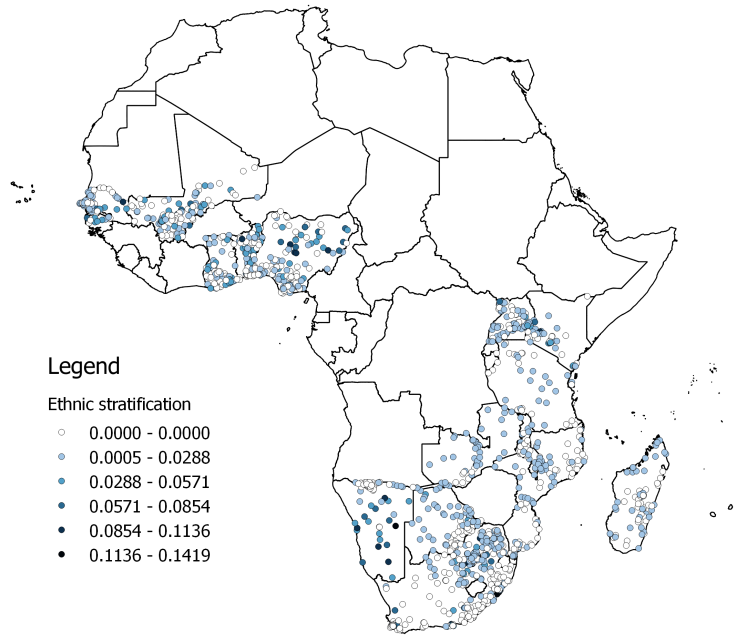




\section{SUMmaRY STATISTICS}

\begin{tabular}{lccccc}
\hline \hline Variable & Obs. & Mean & Std.Dev. & Min. & Max. \\
\hline Round 5: & & & & & \\
\hline Trust in relatives & 21,544 & 0.826 & 0.379 & 0 & 1 \\
Trust in neighbors & 21,521 & 0.607 & 0.488 & 0 & 1 \\
Trust in other acquaintances & 21,475 & 0.416 & 0.493 & 0 & 1 \\
Trust in local assembly & 19,236 & 0.493 & 0.500 & 0 & 1 \\
Ethnic stratification (S) & 21,579 & 0.007 & 0.013 & 0 & 0.098 \\
Fractionalization (F) & 21,579 & 0.103 & 0.147 & 0 & 0.696 \\
Wealth inequality (G) & 21,579 & 0.083 & 0.047 & 0 & 0.390 \\
Average wealth $(\mu)$ & 21,579 & 0.341 & 0.069 & 0.094 & 0.738 \\
\hline Round 3: & & & & & \\
Trust in own ethnic group & 11,833 & 0.550 & 0.498 & 0 & 1 \\
Trust in other ethnic group & 11,710 & 0.420 & 0.494 & 0 & 1 \\
Higher trust in own group & 11,683 & 0.263 & 0.440 & 0 & 1 \\
Ethnic stratification (S) & 11,860 & 0.013 & 0.022 & 0 & 0.142 \\
Fractionalization (F) & 11,860 & 0.101 & 0.149 & 0 & 0.639 \\
Wealth inequality (G) & 11,860 & 0.252 & 0.103 & 0 & 0.750 \\
Average wealth $(\mu)$ & 11,860 & 0.202 & 0.058 & 0 & 0.551 \\
\hline \hline
\end{tabular}




\section{Robustness tests}

- Using index based primarily on ethnicity instead of language

- Compute ethnolinguistic distances using the formula by Fearon (2003) instead of Putterman and Weil (2010)

- Measure economic distances using the perception-based lived poverty index instead of wealth index

- Controlling for respondent's generalized trust and for cluster-level average of generalized trust

- Controlling for trustworthiness of people and local political institutions

- Use categorical answers to trust questions to build variables that can take values 0 ("not at all"), 1 ("just a little"), 2 ("somewhat"), or 3 ("a lot")

- Estimate Probit maximum likelihood models instead of linear probability models

- Round 5: sample restricted to countries from round 3

Most ROBUSTNESS TESTS SUPPORT THE GENERAL PATTERN OF OUR RESULTS. 


\section{AXIOMATIC CHARACTERIZATION OF ETHNIC STRATIFICATION}

We can show that (2) satisfies the following axioms

- Co-directionality by wealth creation or transfer

- Bi-polarization by wealth transfer

- Co-directionality by linguistic change 


\section{CO-DIRECTIONALITY BY WEALTH CREATION OR TRANSFER}

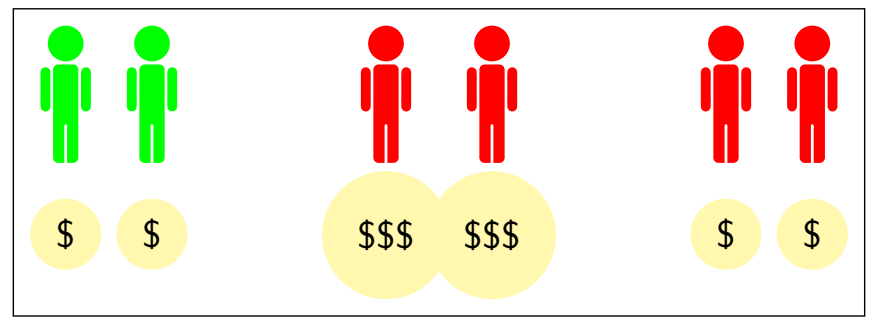




\section{Co-DIRECTIONALITY BY WEALTH CREATION OR TRANSFER}

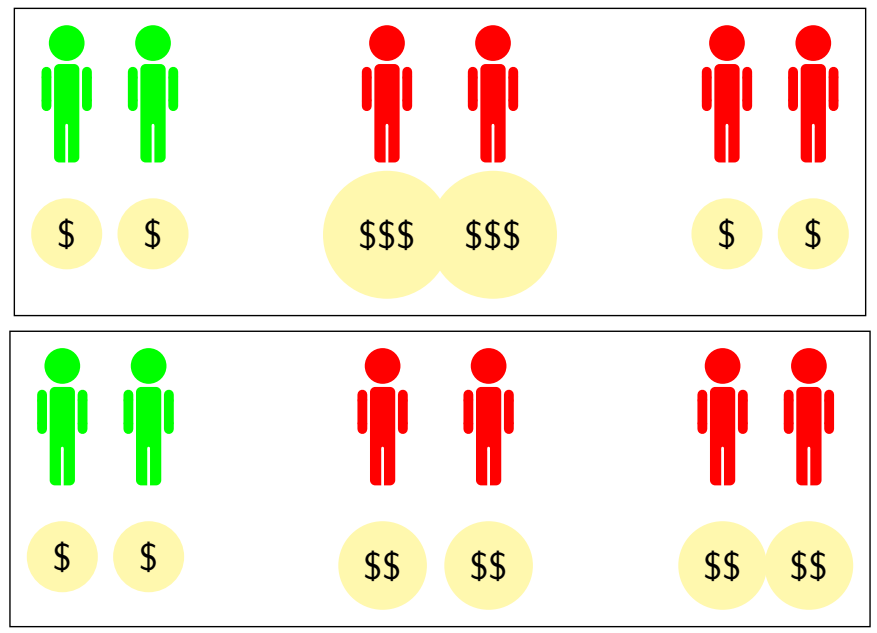




\section{Bi-Polarization By WEALth transfer}

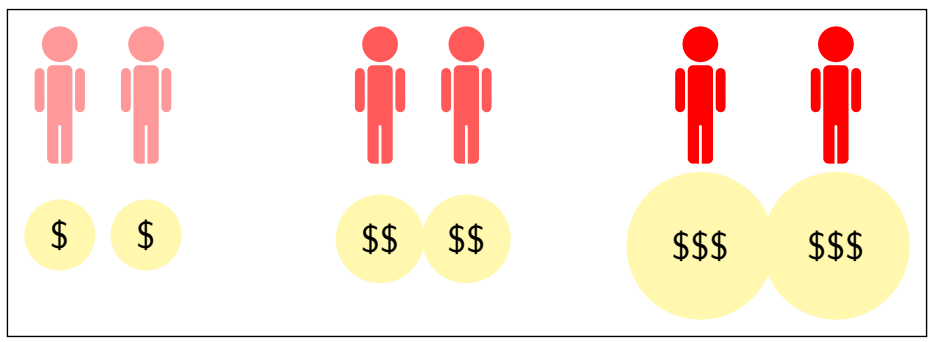




\section{Bi-Polarization By WEALth transfer}

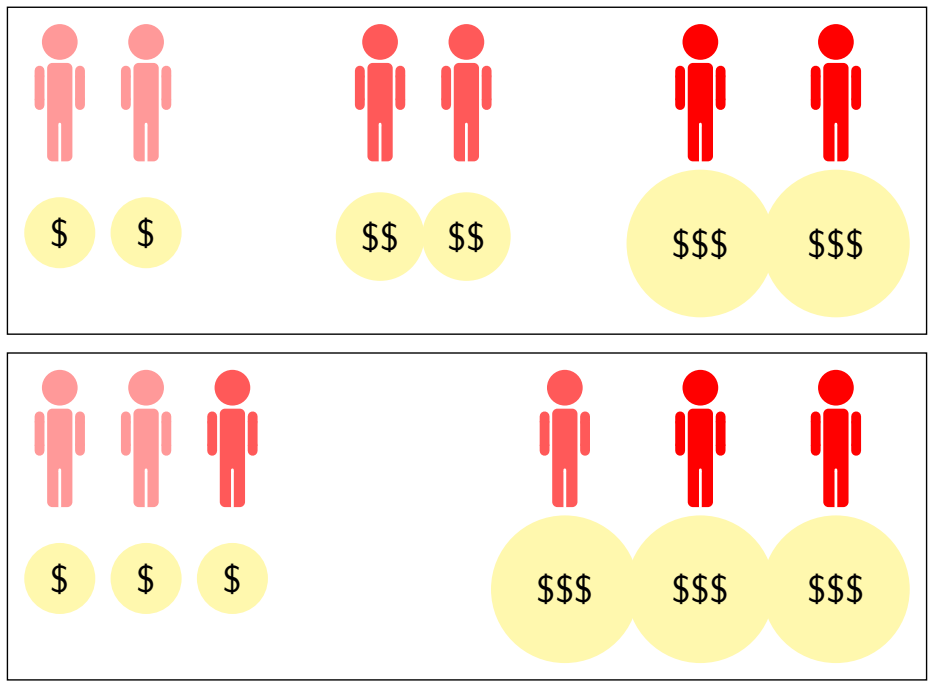




\section{Co-DIRECTIONALITY BY LINGUISTIC CHANGE}

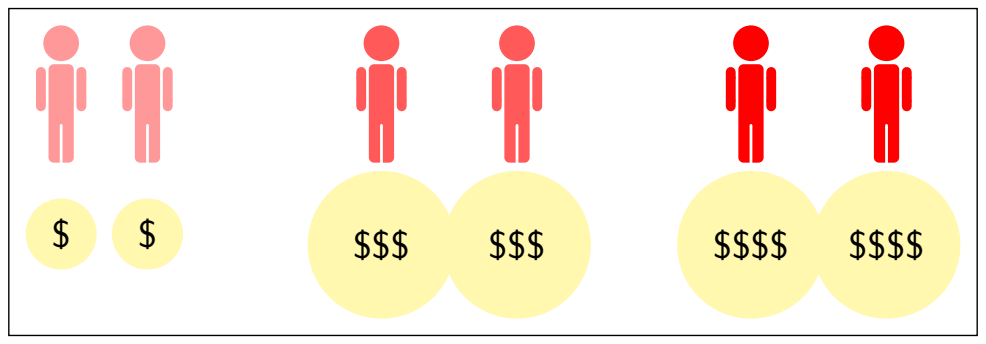




\section{Co-DIRECTIONALITY BY LINGUISTIC CHANGE}

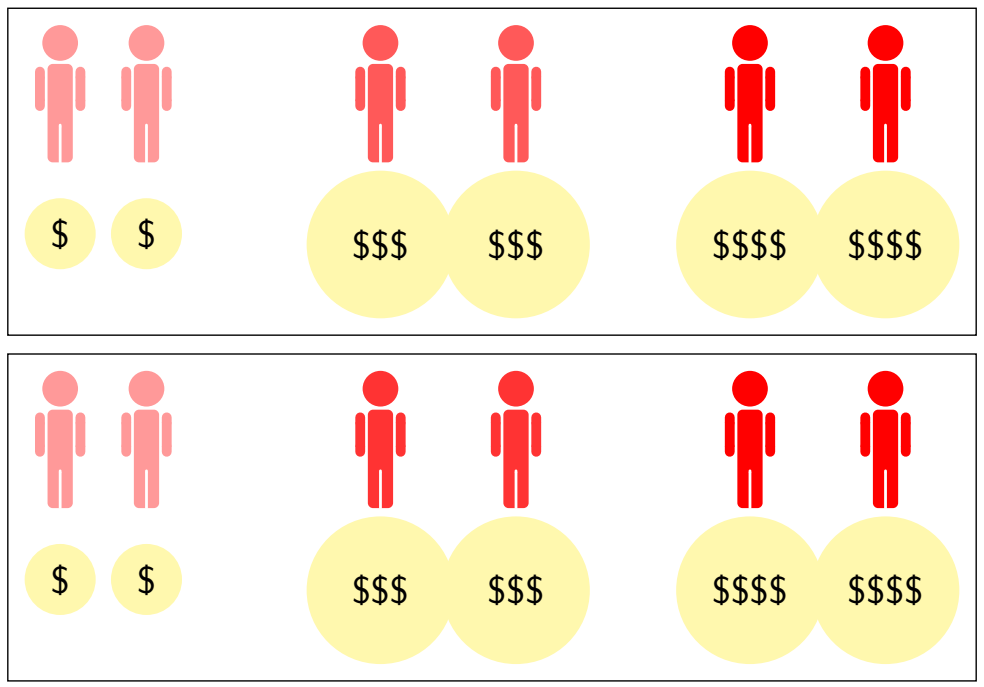

